\title{
Các nhân tố tác động đến quyết định tham gia lấy ý kiến đánh giá học phần của sinh viên Đại học Cần Tho
}

\section{Factors affecting the decision to participate in the evaluation of modules of the students at Can Tho University}

\author{
Hà Mỹ Trang ${ }^{1 *}$, Thạch Keo Sa Ráte ${ }^{1}$, Phan Thị Ánh Nguyệt ${ }^{1}$, Trần Thị Thủy Tiên ${ }^{1}$ \\ ${ }^{1}$ Trường Đại học Cần Thơ, Việt Nam \\ *Tác giả liên hệ, Email: hmtrang@ctu.edu.vn
}

\begin{tabular}{l} 
THÔNG TIN \\
\hline $\begin{array}{l}\text { DOI:10.46223/HCMCOUJS } \\
\text { soci.vi.16.1.1447.2021 }\end{array}$ \\
Ngày nhận: 25/02/2021 \\
Ngày nhận lại: 24/03/2021 \\
Duyệt đăng: 09/04/2021
\end{tabular}

Tù khóa:

đảm bảo chất lượng, đánh giá hoạt động giảng dạy, tỷ lệ phản hồi, mô hình chấp nhận và sử dụng công nghệ, đánh giá trực tuyến

\section{TÓM TẮT}

Công tác lấy ý kiến đánh giá học phần của sinh viên chính quy về hoạt động giảng dạy của giảng viên được xem là một phương pháp hiệu quả làm cơ sở đề xuất các giải pháp đảm bảo chất lượng giáo dục tại các trường đại học. Xem xét tại trường Đại học Cần Thơ, công tác đánh giá này được thực hiện dưới hình thức đánh giá trực tuyến, tuy nhiên tỷ lệ phản hồi của sinh viên là khá thấp và có xu hướng giảm. Nghiên cứu áp dụng phương pháp thống kê mô tả, phương pháp phân tích nhân tố khám phá và phương pháp hồi quy logistic nhằm tìm ra các nhân tố ảnh hưởng đến quyết định tham gia lấy ý kiến đánh giá học phần của sinh viên, trên cơ sở đó đề xuất một số giải pháp nhằm cải thiện công tác lấy ý kiến đánh giá của sinh viên về hoạt động giảng dạy của giảng viên tại trường. Với số lượng sinh viên tham gia khảo sát là 395 sinh viên, nghiên cứu đã xác định được bốn nhân tố ảnh hưởng đến quyết định tham gia đánh giá học phần trực tuyến là khóa học, nhân tố an toàn bảo mật cho người dùng, nhân tố lợi ích có được khi thực hiện đánh giá và cảm nhận về tính dễ thực hiện có ảnh hưởng tích cực làm tăng xác suất tham gia đánh giá học phần của sinh viên. Từ kết quả nghiên cứu, rà soát các quy định hiện hành và tham khảo ý kiến chuyên gia, nghiên cứu đề xuất một số giải pháp như cải thiện cơ sở hạ tầng kỹ thuật, tăng cường thông tin truyền thông, nâng cao nhận thức đối với sinh viên từ đó tăng tỷ lệ phản hồi của sinh viên đối với hoạt động đánh giá học phần trực tuyến.

\section{ABSTRACT}

Student assessment of lecturers' teaching is an effective method to provide a basis for solutions to ensure the training's quality at universities. At Can Tho University, this assessment has been carried out in the form of an online assessment; however, the student's response rates are quite low and tend to decrease over the years. Descriptive statistics, the exploratory factor analysis method, and logistic regression were used to identify the factors that affect students' participation in this 


\section{Keywords:}

quality assurance, evaluation of teaching, response rates, unified theory of acceptance and use of technology, online evaluation assessment, on which basis proposing some solutions to improve this students' assessment at Can Tho University. With the number of students participating in the survey of 395 students, the research has identified four factors that affect students' decision to participate in the assessment: courses, safety and security for users, benefits when making the assessment, and feeling easy of implementation have the effect of increasing student participation probability. Relying on the results, checking current regulations, and consultation with experts, the study has proposed solutions such as improving technical infrastructure, enhancing information and communication activities, raising awareness for students to increase the students' response rates to this assessment activity.

\section{Giới thiệu}

Hiện nay, việc nâng cao chất lượng Giáo Dục Đại Học (GDĐH) là một vấn đề tối quan trọng đối với các trường đại học nhằm đáp ứng nhu cầu về chất lượng nguồn nhân lực của thị trường lao động. Đã có nhiều mô hình quản lý chất lượng được xây dựng nhằm đáp ứng mục tiêu nâng cao chất lượng giáo dục, cụ thể như mô hình OME (Organizational Elements Models), mô hình đánh giá chất lượng của Mỹ, mô hình BS 5750, mô hình ISO 9001:2000, mô hình kiểm định chất lượng theo tiêu chuẩn AUN_QA. Điểm chung của các mô hình đều đề cao vai trò và ý kiến phản hồi của người học trong việc nâng cao chất lượng đào tạo. Khẳng định này cũng phù hợp với kết quả từ các nghiên cứu đi trước như Dommeyer, Baum, Hanna, và Chapman (2004); Nair, Commission, và Mertova (2015). Cùng với sự phát triển của công nghệ hoạt động lấy ý kiến phản hồi của người học đã chuyển dần từ đánh giá bằng giấy sang hình thức đánh giá trực tuyến với nhiều điểm tích cực như tiết kiệm thời gian, tiết kiệm chi phí nhưng tỷ lệ phản hồi thấp và giảm dần là thách thức chung của hình thức đánh giá này.

Tại Việt Nam, qua quá trình hình thành và phát triển, mô hình kiểm định chất lượng theo tiêu chuẩn AUN_QA được sử dụng và triển khai rộng rãi tại các cơ sở GDĐH. Mô hình đảm bảo chất lượng bên trong của AUN_QA nhấn mạnh vai trò của các bên liên quan mà cụ thể là vai trò về sự tham gia của sinh viên trong đảm bảo chất lượng giáo dục. Đóng góp vào sự hoàn thiện của hoạt động kiểm định chất lượng và đảm bảo quy chế dân chủ trong cơ sở GDĐH Bộ Giáo dục và Đào tạo đã ban hành Công văn số 1276/BGDĐT ngày 20/02/2008 về "Hướng dẫn tổ chức lấy ý kiến phản hồi từ người học về hoạt động giảng dạy của giảng viên". Tuy nhiên, tại trường Đại học Cần Thơ, công tác lấy ý kiến của sinh viên về hoạt động giảng dạy của giảng viên hiện nay còn một số tồn tại đã cản trở quyết định tham gia lấy ý kiến đánh giá học phần của sinh viên. Kết quả dẫn đến tỷ lệ tham gia của sinh viên vào hoạt động này là hạn chế và có xu hướng giảm. Nhằm tìm ra các nhân tố tác động đến quyết định tham gia lấy ý kiến đánh giá học phần của sinh viên thì nghiên cứu này có vai trò rất quan trọng mà chưa có nghiên cứu trong nước nào thực hiện trước đó. Nghiên cứu góp phần xác định các nhân tố ảnh hưởng đến quyết định tham gia lấy ý kiến đánh giá học phần và đề ra các giải pháp phù hợp cho các trường đại học trong quá trình nâng cao chất lượng đào tạo.

\section{Cơ sở lý thuyết và mô hình nghiên cứu}

\subsection{Cơ sở lý thuyết}

\subsubsection{Khái niệm về chất lượng giáo dục đại học}

Có nhiều khái niệm khác nhau về chất lượng GDĐH, theo Schindler, Puls-Elvidge, 
Welzant, và Crawford (2015) chất lượng GDĐH là sự đáp ứng các tuyên bố về tầm nhìn sứ mạng, là việc hoàn thành các mục tiêu đã công bố của các cơ sở GDĐH. Đồng thời, chất lượng GDĐH còn được xem xét là sự cải tiến trong hoạt động đào tạo, cũng như trách nhiệm giải trình của các bên liên quan (Cheng \& Tam, 1997; Harvey \& Knight, 1996). Tại Việt Nam, chất lượng GDĐH là sự đáp ứng các yêu cầu của Luật giáo dục và các quy định của nhà nước về GDĐH (Khoản 1, Điều 2, Thông tư 61/TT-BGDĐT, 2012). Chất lượng GDĐH còn được xem xét thông qua việc hoàn thiện chuẩn đầu ra, phản ánh hiệu quả đầu tư trong lĩnh vực giáo dục. Đồng thời, chất lượng giáo dục "là sự đáp ứng của sản phẩm đào tạo đối với các chuẩn mực và tiêu chí đã được xác định” (K. D. Nguyen \& Pham, 2003).

\subsubsection{Hoạt động lấy ý kiến phản hồi tì̀ người học}

Hoạt động lấy ý kiến phản hồi từ người học hay còn gọi là đánh giá học phần là một phương pháp quản trị giáo dục hiệu quả được sử dụng rộng rãi trên toàn thế giới (Campbell \& Wende, 2000; Ubong \& Okpor, 2019). Hoạt động lấy ý kiến phản hồi từ người học là công cụ quan trọng góp phần nâng cao chất lượng giáo dục (Ryan, 2015; Young, Joines, Standish, \& Gallagher, 2017). Nếu hoạt động này được thực hiện tốt sẽ đem lại lợi ích cho các bên liên quan. Cụ thể, kết quả đánh giá học phần sẽ là cơ sở giúp các trường cải tiến chương trình đào tạo, là thước đo đánh giá năng lực của giảng viên, cung cấp thông tin hữu ích để giảng viên tự đánh giá và hoàn thiện bản thân, từ đó nâng cao năng lực giảng dạy, đồng thời giúp sinh viên thể hiện được quan điểm cá nhân một cách khách quan nhất (Nowell, Gale, \& Handley, 2010; Standish, Joines, Young, \& Gallagher, 2018).

Với sự phát triển của công nghệ thông tin, việc tiếp nhận và thu thập ý kiến phản hồi của sinh viên đã chuyển đổi từ hình thức đánh giá bằng giấy sang đánh giá trực tuyến (Ballantyne, 2003; Ravelli, 2000). Việc chuyển đổi này mang lại nhiều điểm tích cực cho các cơ sở GDĐH như tiết kiệm thời gian cho người học, người quản lý trong việc thu thập và tổng hợp dữ liệu. Tuy nhiên, tỷ lệ phản hồi thấp sau khi chuyển đổi vẫn là một thách thức quan trọng đối với các trường, vì vậy đòi hỏi cần có những chính sách kịp thời nhằm gia tăng sự tham gia của sinh viên vào hoạt động đánh giá học phần.

\subsubsection{Các lý thuyết và mô hình nghiên cưu về quyết định tham gia}

Có nhiều lý thuyết và mô hình nghiên cứu các yếu tố ảnh hưởng đến quyết định của con người. Các quyết định này không chỉ dừng lại ở việc chấp nhận một sản phẩm mới, mà còn là chấp nhận một quy trình, một công nghệ mới, hay một phương pháp mới. Trên cơ sở tổng hợp từ các nghiên cứu đi trước đề tài giới thiệu các mô hình tiêu biểu sau:

Được phát triển từ năm 1962, lý thuyết lan truyền sự đổi mới của Rogers (1995) nghiên cứu làm thế nào sự tiến bộ của công nghệ có thể lan tỏa trong xã hội. Lý thuyết nghiên cứu bốn nhóm yếu tố ảnh hưởng đến quyết định tiếp nhận sự đổi mới gồm "đặc điểm con người", "kênh truyền thông", "thời gian" và "hệ thống xã hội”. Khi một ý tưởng mới được biết đến nó thu hút sự quan tâm của con người. Theo thời gian các ý tưởng này được mọi người tiếp nhận tùy thuộc vào đặc điểm cá nhân, ảnh hưởng từ các kênh thông tin truyền thông và ảnh hưởng từ xã hội từ đó hình thành quyết định chấp nhận sự đổi mới.

Dựa trên nền tảng của lý thuyết lan truyền sự đổi mới, Fishbein và Ajzen (1981) đã xây dựng mô hình lý thuyết hành động hợp lý. Mô hình này khẳng định quyết định của con người chịu sự ảnh hưởng của ý định hành vi, đo lường bởi "thái độ đối với hành vi" và "chuẩn chủ quan". Trong đó, thái độ hành vi là niềm tin của con người đối với công nghệ mới, đồng thời các yếu tố từ bên ngoài như tác động từ gia đình, xã hội hay còn gọi là chuẩn chủ quan cũng góp phần không nhỏ gây ra ý định thực hiện hành vi. Nhưng điểm hạn chế của mô hình này là nhấn 
mạnh vai trò của hành vi được kiểm soát bởi lí trí, còn đối với các hành vi không hợp lý hay các hành vi chịu sự tác động từ các yếu tố bên ngoài thì mô hình chưa xem xét tới.

Để giải quyết hạn chế của mô hình lý thuyết hành động hợp lý, Ajzen (1991) đề xuất mô hình lý thuyết hành vi hoạch định gồm ba nhóm yếu tố thái độ đối với hành vi, chuẩn chủ quan và nhận thức về kiểm soát hành vi ảnh hưởng đến ý định hành vi và quyết định hành vi của con người.

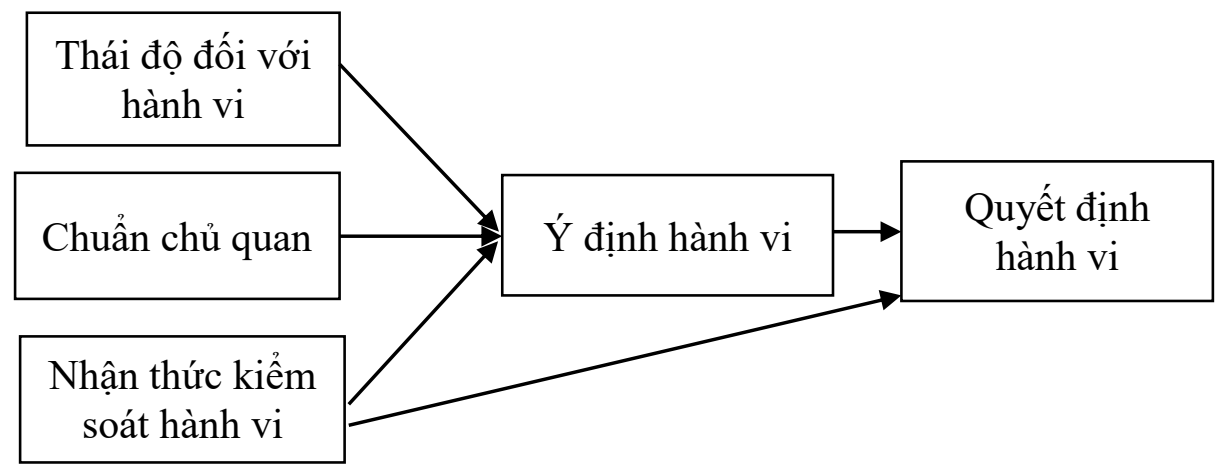

Hình 1. Mô hình lý thuyết hành hành vi hoạch định của Ajzen

Nguồn: Ajzen (1991)

Qua Hình 1 cho thấy bên cạnh "thái độ đối với hành vi” và “chuẩn chủ quan” thì "nhận thức kiểm soát hành vi” được bổ sung vào mô hình lý thuyết hành động hợp lý, nhân tố này được xem xét là các điều kiện thuận lợi và các cơ hội khách quan được tạo ra ảnh hưởng đến ý định hành vi và quyết định hành vi của con người.

Ngoài những mô hình trên thì mô hình chấp nhận và sử dụng công nghệ (Unified Theory of Acceptance and Use of Technology - UTAUT) của Venkatesh, Morris, Davis, và Davis (2003) là một bước tiến vượt bậc trong lĩnh vực nghiên cứu quyết định hành vi có sử dụng công nghệ của con người. Mô hình UTAUT khắc phục và hoàn thiện những hạn chế của các mô hình đi trước và được minh họa như sau:

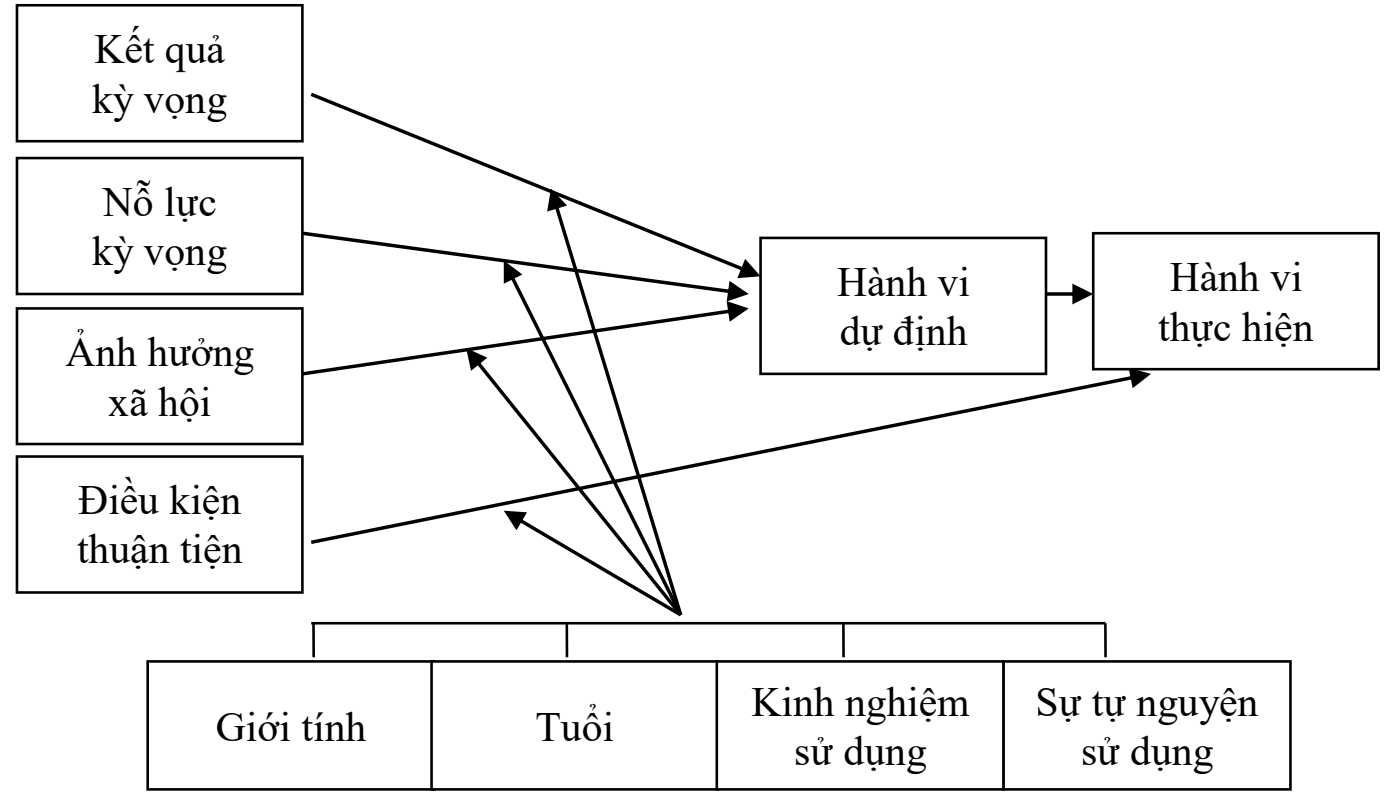

Hình 2. Mô hình chấp nhận và sử dụng công nghệ của Venkatesh và cộng sự (2003)

Nguồn: Venkatesh và cộng sự (2003) 
Theo Venkatesh và cộng sự (2003) thì có nhiều yếu tố ảnh hưởng đến hành vi dự định và hành vi thực hiện của con người. Thứ nhất, "kết quả kỳ vọng" được đo lường qua niềm tin về các lợi ích mà cá nhân sẽ đạt được khi chấp nhận công nghệ mới hoặc sử dụng hệ thống mới. Thứ hai, "nỗ lực kỳ vọng” được xem xét là mức độ dễ dàng cho cá nhân khi tiếp cận công nghệ mới. Thứ ba, “ảnh hưởng xã hội” đo lường mức độ mà con người sẽ quyết định sử dụng hệ thống mới dưới sự ảnh hưởng của những người xung quanh. Thứ tư, “điều kiện thuận lợi” là các chỉ tiêu về cơ sở hạ tầng kỹ thuật hay quy trình giúp người dùng dễ dàng tiếp cận với công nghệ mới. Thứ năm, hành vi dự định đo lường mức độ con người sẵn lòng chấp nhận công nghệ mới, hành vi dự định có vai trò quan trọng dẫn đến việc thực hiện hành vi của con người. Các biến nhân khẩu học cũng được đưa vào mô hình nhằm giải thích sự khác biệt về đặc tính nhân khẩu học ảnh hưởng đến việc thực hiện hành vi của con người.

\subsection{Mô hình nghiên cúu của đề tài}

Nghiên cứu kế thừa khung phân tích UTAUT của Venkatesh và cộng sự (2003), đồng thời nghiên cứu bổ sung thêm nhân tố "An toàn bảo mật đối với người dùng” với kỳ vọng nhân tố này có ảnh hưởng tích cực đến đến quyết định tham gia lấy ý kiến đánh giá của sinh viên. Theo Ballantyne (2003) tính ẩn danh và bảo mật là nguyên nhân dẫn đến tỷ lệ phản hồi trực tuyến thấp. Cụ thể, tại Đại học Murdoch sinh viên cần cung cấp mã số sinh viên mới có thể thực hiện đánh giá vì vậy tỷ lệ tham gia phản hồi của sinh viên giảm đáng kể so với hình thức truyền thống. Theo Ravelli (2000), Fike, Doyle, và Connelly (2010) khẳng định hoạt động khảo sát trực tuyến nếu đảm bảo về tính an toàn bảo mật sẽ có tác động tích cực giúp sinh viên tự tin tham gia và mạnh dạng phản hồi quan điểm của mình. Vì vậy, "An toàn bảo mật đối với người dùng" được tác giả đề xuất đưa vào mô hình là hoàn toàn có cơ sở. Mô hình nghiên cứu của đề tài được trình bày như sau:

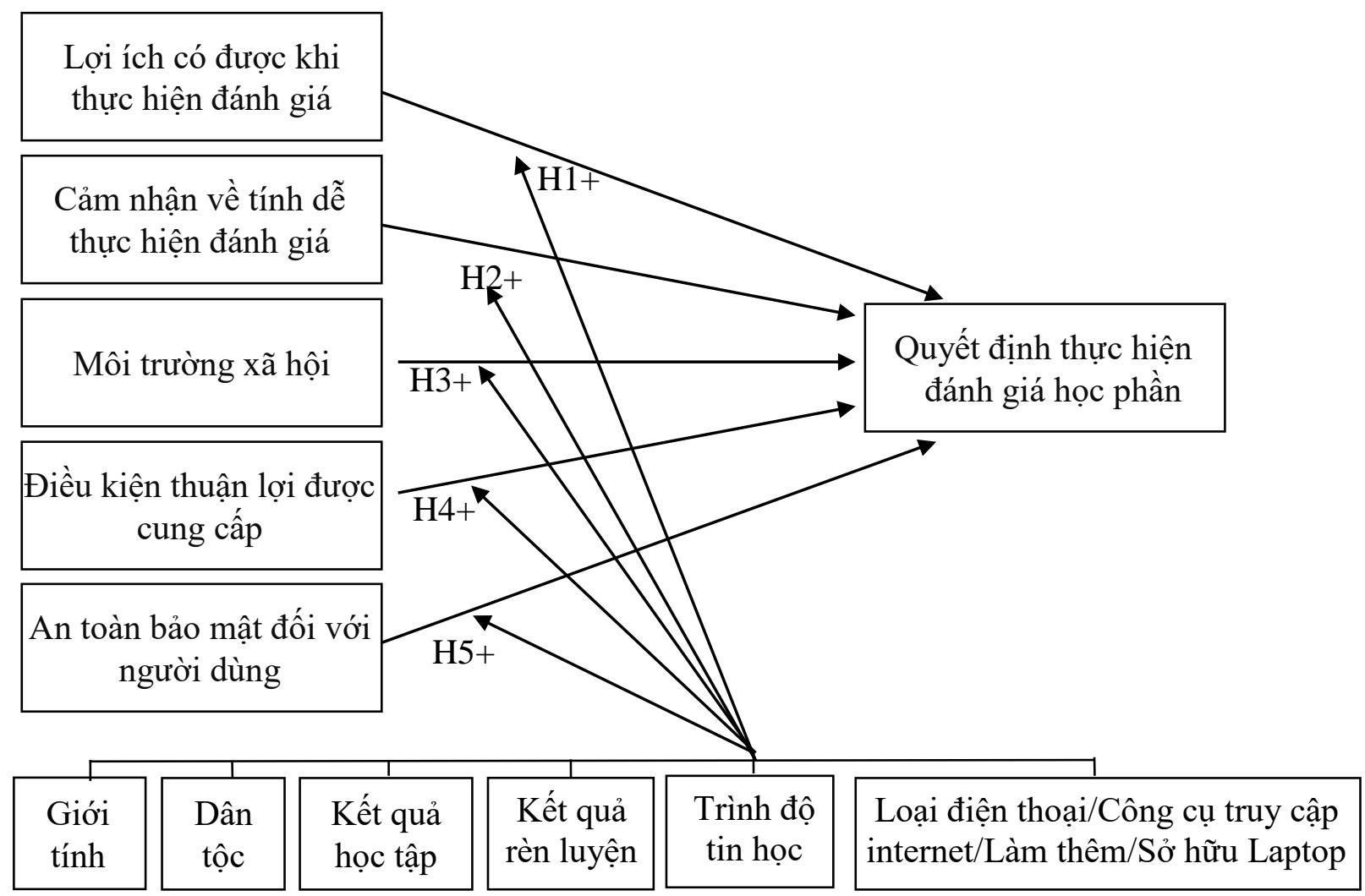

Hình 3. Các nhân tố ảnh hưởng đến quyết định tham gia đánh giá học phần 
Đối với biến phụ thuộc “Quyết định thực hiện đánh giá học phần” gọi tắt là biến Y là một biến Dummy với 02 giá trị được xem xét là $\mathrm{Y}=1$ sinh viên có thực hiện đánh giá học phần và $\mathrm{Y}$ $=0$ sinh viên không thực hiện đánh giá học phần. Các nhân tố ảnh hưởng đến quyết định thực hiện đánh học phần sử dụng thang đo Likert 5 mức độ từ "Rất không đồng ý" đến "Rất đồng ý". Nghiên cứu xây dựng giả thuyết thống kê và thang đo như sau:

H1.1: Lợi ích có được khi thực hiện đánh giá học phần có ảnh hưởng tích cực đến quyết định thực hiện đánh giá học phần của sinh viên

Lợi ích có được khi thực hiện đánh giá học phần $(\mathrm{LI})$ thể hiện mức độ sinh viên tin rằng họ sẽ nhận được những lợi ích khi tham gia đánh giá học phần trực tuyến. Theo Adams (2010) và Ravelli (2000) sinh viên tin rằng đánh giá của họ sẽ "giúp giảng viên cải thiện chất lượng giảng dạy" và "đánh giá học phần trực tuyến rất hữu ích với họ” từ đó họ sẽ tiếp nhận phương pháp giảng dạy tốt hơn. Ngoài ra, đánh giá trực tuyến sẽ giúp sinh viên "tiết kiệm thời gian và chi phí của bản thân” (Ballantyne, 2003; Fan \& Yan, 2010; Ravelli, 2000).

H1.2: Cảm nhận về tính dễ thưc hiện đánh giá có ảnh huởng tích cực đến quyết định thực hiện đánh giá học phần của sinh viên

Cảm nhận về tính Dễ Thực Hiện đánh giá (DTH) thể hiện mức độ kỳ vọng của sinh viên cho rằng họ dễ dàng tìm hiểu và thực hiện đánh giá học phần, hoạt động này phù hợp với năng lực của họ và nội dung trả lời đánh giá là đơn giản. Theo Ravelli (2000), Sax, Gilmartin, và Bryant (2011) nếu tìm hiểu và thực hiện đánh giá học phần là đơn giản đối với sinh viên thì sinh viên sẽ tham gia đánh giá học phần tích cực hơn. Đồng thời, nghiên cứu của Standish và cộng sự (2018), Ballantyne (2003) cũng phản ánh kỳ vọng của sinh viên về nội dung bảng câu hỏi dễ̂ hiểu, thời gian tổ chức linh hoạt sẽ quyết định nên hành vi tham gia của sinh viên.

H1.3: Môi truòng xã hội có ảnh hưởng tích cực đến quyết định thực hiện đánh giá học phần của sinh viên

Môi Trường Xã Hội (MTXH) thể hiện mức độ nhận thức của sinh viên nghĩ rằng những người xung quanh họ đồng thuận và khuyến khích họ thực hiện đánh giá học phần, từ đó ảnh hưởng đến động cơ và hành vi thực hiện của sinh viên. Theo Goodman, Anson, và Belcheir (2015), Cummings và Ballantyne (1999) sự nhắc nhở từ phía giảng viên có vai trò quan trọng ảnh hưởng đến hành vi thực hiện của sinh viên. Ngoài ra, Venkatesh và cộng sự (2003), Porter và Umbach (2006) đề cao vai trò của bạn bè xung quanh trong việc nhắc nhở và lôi kéo sinh viên thực hiện đánh giá học phần. Đồng thời, khi học tập trong môi trường mà mọi người đều thực hiện đánh giá cũng tạo nên hiệu ứng lan tỏa đến bản thân của sinh viên (Cummings \& Ballantyne, 1999; Goodman et al., 2015).

H1.4: Điều kiện thuận lợi được cung cấp có ảnh hương tích cực đến quyết định thực hiện đánh giá hoc phần của sinh viên

Điều Kiện Thuận Lợi được cung cấp (ĐKTL) phản ánh mức độ sinh viên được cung cấp đầy đủ cơ sở hạ tầng kỹ thuật như máy tính, sự nhắc nhở từ hệ thống và nhận được hỗ trợ kịp thời để thực hiện đánh giá học phần. Cụ thể, hệ thống máy chủ ổn định, máy tính hiện đại, mạng lưới wifi rộng khắp, đường truyền nhanh chóng và được hỗ trợ sự cố 24/24 sẽ có tác động tích đến quyết định đánh giá học phần của sinh viên (Cummings \& Ballantyne, 1999; Ravelli, 2000; Standish et al., 2018).

H1.5: An toàn bảo mật đối với người dùng có ảnh hương tích cục đến quyết định thực hiện đánh giá học phần của sinh viên

An Toàn Bảo Mật đối với người dùng (ATBM) là nhân tố mới được tác giả bổ sung vào 
mô hình UTAUT của Venkatesh và cộng sự (2003). An toàn bảo mật phản ánh mức độ sinh viên tin rằng sự tham gia và ý kiến của họ khi đánh giá học phần sẽ được giữ bí mật tuyệt đối. Theo Standish và cộng sự (2018), Ballantyne (2003) sinh viên sẽ thực hiện đánh giá trực tuyến nếu cảm nhận hệ thống đánh giá có tính bảo mật cao. Đồng thời, theo Sax và cộng sự (2011) khi hệ thống đánh giá có tính ẩn danh và kết quả đánh giá được giữ bí mật tuyệt đối sẽ phản ánh mức độ tham gia đánh giá trực tuyến của sinh viên ngày càng tích cực hơn. Các yếu tố này cũng được khẳng định trong nghiên cứu trước đó của Ravelli (2000).

Nghiên cứu còn xem xét đến các yếu tố nhân khẩu của sinh viên như giới tính (gtinh), dân tộc (dtoc), nơi ở (noio), khóa học (khoa), khoa đào tạo (colledge) kết quả học tập (hocluc), kết quả rèn luyện (renluyen), trình độ tin học (tinhoc), loại điện thoại đang dùng (dthoai), công cụ truy cập internet (congcu), trình trạng làm thêm (exjob) và tình trạng sở hữu laptop (laptop) nhằm đánh giá sự khác biệt về các đặc tính nhân khẩu học giữa nhóm sinh viên quyết định thực hiện đánh giá và nhóm sinh viên không thực hiện đánh giá. Các yếu tố nhân khẩu học này được đề cập phần lớn trong nghiên cứu của Spencer và Schmelkin (2010), Norris và Moon (2005), Cummings và Ballantyne (1999). Theo Porter và Umbach (2006) giới tính và dân tộc ảnh hưởng đến quyết định thực hiện đánh giá học phần. Cụ thể, sinh viên nữ có xu hướng tham gia đánh giá nhiều hơn sinh viên nam, sinh viên da màu có xu hướng tham gia đánh giá ít hơn sinh viên da trắng. Theo Eng, Ibrahim, và Shamsuddin (2015), Franklin và Theall (1992) cho thấy kết quả học tập cũng ảnh hưởng đến hành vi đánh giá của sinh viên. Sinh viên có kết quả học tập khá, giỏi thường tích cực hơn trong các hoạt động, do đó xác suất tham gia đánh giá trực tuyến sẽ cao hơn. Đặc biệt, theo Standish và cộng sự (2018) kết luận việc thực hiện đánh giá bằng điện thoại thông minh, kết hợp cài đặt các ứng dụng điện thoại cũng khuyến khích sinh viên tham gia thực hiện đánh giá các bảng câu hỏi trực tuyến.

\section{Phương pháp thu thập dữ liệu và phương pháp nghiên cứu}

\subsection{Phưong pháp thu thập dĩ liệu}

Nghiên cứu sử dụng dữ liệu thứ cấp thu thập từ trường Đại học Cần Thơ và dữ liệu sơ cấp thông qua bảng câu hỏi phỏng vấn trực tiếp các sinh viên tại trường Đại học Cần Thơ. Đồng thời, nghiên cứu phỏng vấn trực tiếp chuyên gia nhằm có cơ sở đề ra giải pháp cải thiện hoạt động đánh giá học phần trực tuyến trong sinh viên.

\subsection{Phưong pháp chọn mẫu}

Nhằm đảm bảo tính đại diện cho cỡ mẫu đề tài sử dụng phương pháp chọn mẫu ngẫu nhiên phân tầng theo khoa đào tạo. Số lượng cỡ mẫu được xác định bằng công thức:

$$
n=\frac{N}{1+N * e^{2}}
$$

Trong đó: $\mathrm{n}$ là số lượng cỡ mẫu;

$\mathrm{N}$ là tổng số sinh viên;

e là sai số tối đa (e = 1 - độ tin cậy), giả sử độ tin cậy $95 \%$.

Nghiên cứu hướng đến ba khoa có số lượng sinh viên đông nhất trường Đại học Cần Thơ là khoa Kinh tế với tổng số 4.831 sinh viên, khoa Công nghệ với tổng 4.937 sinh viên, khoa Nông nghiệp với tổng số 3.300 sinh viên. Từ công thức (1) với độ tin cậy $95 \%$, cỡ mẫu cần nghiên cứu tối thiểu là 389 sinh viên. Vậy số lượng cỡ mẫu đề tài sẽ tiến hành phỏng vấn là 395 sinh viên là phù hợp và nghiên cứu sẽ phân tầng số lượng cỡ mẫu theo khoa đào tạo, lần lượt là 
khoa Kinh tế 146 sinh viên, khoa Công nghệ 149 sinh viên, khoa Nông nghiệp 100 sinh viên ${ }^{1}$.

\subsection{Phuơng pháp phân tích}

Phương pháp thống kê mô tả được sử dụng để phân tích, trình bày và giải thích dữ liệu thông qua các giá trị thống kê, qua sơ đồ và biểu đồ nhằm thể hiện được thực trạng đánh giá học phần trực tuyến của sinh viên chính quy, đồng thời trình bày và mô tả về mẫu khảo sát thông qua các giá trị thống kê như giá trị trung bình, tần số.

Phương pháp phân tích định tính nhằm tổng hợp, phân loại và đánh giá điểm tích cực, điểm hạn chế về thực trạng đánh giá học phần của sinh viên chính quy về hoạt động giảng dạy của sinh viên tại trường Đại học Cần Thơ.

Kiểm định Chi-bình phương được thực hiện nhằm kiểm định mối liên hệ giữa các biến định danh - định danh hoặc định danh - thứ bậc (Hoang \& Chu, 2008), với giả thuyết $\mathrm{H}_{0}$ là không có sự khác biệt về các yếu tố nhân khẩu học với quyết định đánh giá học phần của nhóm sinh viên có quyết định tham gia đánh giá và nhóm sinh viên không tham gia đánh giá.

Kiểm định Cronbach's Alpha dùng để đánh giá mức độ tương quan chặt chẽ giữa các biến quan sát trong cùng một nhân tố được đưa vào mô hình nghiên cứu. Theo D. T. Nguyen (2013) hệ số Cronbach's Alpha có hệ số biến thiên trong khoản $[0,1]$, hệ số này càng cao thì càng tốt nhưng nếu vượt ngưỡng 0,95 thì có hiện tượng trùng lắp trong thang đo. Theo Hoang và Chu (2008) hệ số Cronbach' Alpha từ 0,6 trở lên là đủ điều kiện, từ $0,7-0,8$ là thang đo sử dụng tốt và trên 0,8 là thang đo rất tốt, nếu hệ số tương quan biến tổng 0,3 được đánh giá là không đạt và sẽ bị loại khỏi mô hình.

Phương pháp phân tích nhân tố khám phá (Exploratory Factor Analysis - EFA) nếu như kiểm định Cronbach's Alpha chỉ xem xét các biến quan sát trong cùng một nhân tố thì phương pháp phân tích nhân tố khám phá được xây dựng nhằm xem xét mối quan hệ giữa tất cả các nhân tố được đưa vào mô hình nghiên cứu thông qua các giá trị kiểm định như hệ số KMO (Kaiser Meyer Olkin), Kiểm định Bartlett (Bartlett's test of sphericity), Trị số Eigenvalue, Tổng phương sai trích (Total Variance Explained), Hệ số Factor Loading.

Mô hình hồi quy logistic (Binary Logistic Regression) là phương pháp được sử dụng phổ biến trong trường hợp biến mục tiêu không phải là biến định lượng liên tục. Trong nghiên cứu này mô hình hồi quy logistic dùng để ước lượng xác suất sinh viên sẽ quyết định thực hiện đánh giá học phần chịu sự chi phối bởi các nhân tố được đề cập tại mục 2.2 và các biến nhân khẩu học của đáp viên. Ta có phương trình hồi quy logistic được viết cụ thể như sau:

$$
\begin{aligned}
& \log \left(\frac{p}{1-p}\right)=\alpha+\beta_{1} \text { gtinh }+\beta_{2} \text { dtoc }+\beta_{3} \text { noio }+\beta_{4} \text { khoa }+\beta_{5} \text { colledge }+\beta_{6} \text { hocluc }+ \\
& \beta_{7} \text { renluyen }+\beta_{8} \text { tinhoc }+\beta_{9} \text { dthoai }+\beta_{10} \text { congcu }+\beta_{11} \text { exjob }+\beta_{12} \text { laptop }+\beta_{13} \text { LI }+ \\
& \beta_{13} \text { DTH }+\beta_{14} \text { MTXH }+\beta_{15} \text { ĐTL }+\beta_{16} \text { ATBM }+\varepsilon
\end{aligned}
$$

Trong đó, $\mathrm{p}$ là xác suất để sinh viên có thực hiện có đánh giá học phần và $1-\mathrm{p}$ là xác suất sinh viên không thực hiện đánh giá học phần. Các biến nhân khẩu học là các biến định danh được mã hóa thành các biến giả (biến Dummy) căn cứ vào nội dung bảng câu hỏi. Các biến nhân tố đề xuất dựa trên mô hình nghiên cứu được trình bày ở Hình 3 theo thang đo Likert 5 mức độ và phương pháp phân tích nhân tố khám phá.

\footnotetext{
${ }^{1}$ Nghiên cứu không khảo sát sinh viên năm nhất (Khóa 46) vì đây là nhóm sinh viên chưa thực hiện đánh giá học phần khi tiến hành nghiên cứu này.
} 


\section{Kết quả nghiên cứu}

\subsection{Thống kê công tác lấy ý kiến học phần trục tuyến}

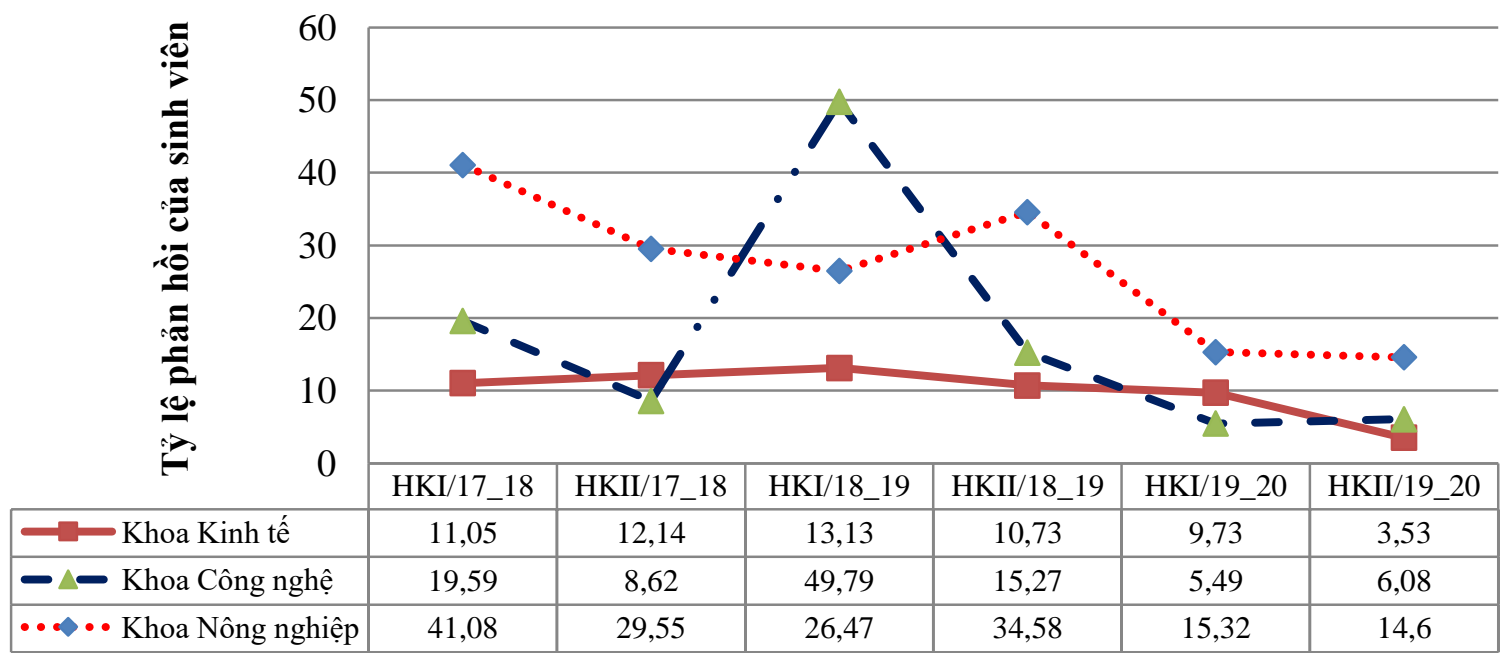

Hình 4. Tỷ lệ tham gia đánh giá học phần của sinh viên

Nguồn: Tổng hợp từ thông tin của Trung tâm quản lý chất lượng, Trường Đại học Cần Thơ (2020)

Tỷ lệ tham gia đánh giá học phần của sinh viên được đo lường qua tổng số phiếu thu về/tổng số phiếu phát ra. Căn cứ vào Hình 4 , nghiên cứu kết luận có sự biến động không đồng đều về tỷ lệ tham gia đánh giá học phần trực tuyến giữa các năm học tại các khoa trong phạm vi nghiên cứu. Tỷ lệ này nhìn chung là khá thấp và có xu hướng giảm dần qua các năm học. Xem xét tại thời điểm học kỳ 2, năm học 2019 - 2020, tỷ lệ phản hồi của sinh viên cao nhất tại khoa Nông nghiệp là $14,6 \%$, tỷ lệ phản hồi tại khoa Công nghệ là $6,08 \%$ và thấp nhất là tỷ lệ phản hồi tại khoa Kinh tế chỉ đạt 3,53\%. Qua số liệu cho thấy tuy tỷ lệ phản hồi của sinh viên tại các khoa đều thấp nhưng khoa Nông nghiệp luôn vượt trội hơn các khoa khác về tỷ lệ phản hồi, điều này có thể lý giải được những ưu điểm của khoa Nông nghiệp khi thực hiện triển khai hoạt động này tại website của khoa. Vì vậy, để tăng tỷ lệ phản hồi của sinh viên đối với hoạt động giảng dạy của giảng viên đòi hỏi nhà trường cần có những thay đổi kịp thời trong hoạt động triển khai và quản lý, từ đó thúc đẩy hoạt động đánh giá học phần đi vào thực chất nhằm nâng cao chất lượng giáo dục tại nhà trường.

\subsection{Kết quả thu thập dĩ liệu sơ cấp}

Qua khảo sát 395 sinh viên tại khoa Kinh tế, khoa Công nghệ, khoa Nông nghiệp, nghiên cứu tiến hành thống kê mô tả các thông tin về mẫu khảo sát như sau:

4.2.1. Số luợng sinh viên tham gia vào mẫu khảo sát phân theo khóa học

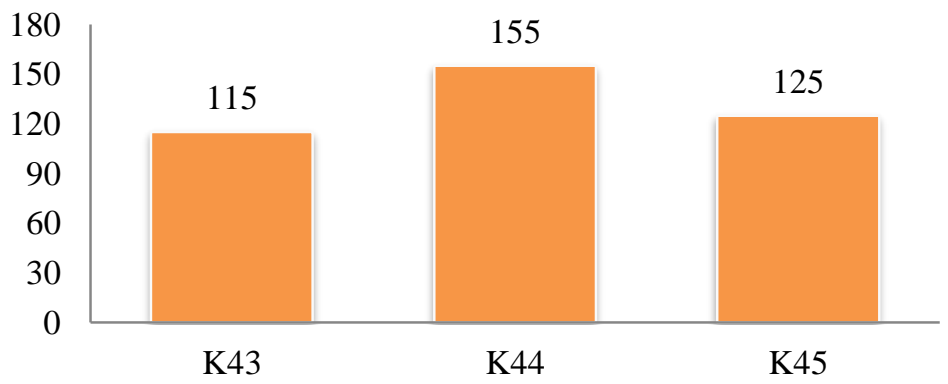

Hình 5. Số lượng sinh viên tham gia vào mẫu khảo sát phân theo khóa 
Nhằm đảm bảo tính đại diện và khách quan cho cỡ mẫu, nghiên cứu sử dụng phương pháp chọn mẫu ngẫu nhiên bằng cách sử dụng hàm Randbetween (bottom, top) trong excel, với số lượng các khóa lần lượt là 115 sinh viên khóa 43 (năm tư), 155 sinh viên khóa 44 (năm ba) và 125 sinh viên khóa 45 (năm hai).

\subsubsection{Tỷ lệ sinh viên tham gia đánh giá học phần trục tuyến}

\section{Bảng 1}

Tỷ lệ sinh viên tham gia đánh giá học phần trực tuyến

\begin{tabular}{|c|c|c|c|}
\hline & Số sinh viên & Tỷ lệ (\%) & Ghi chú \\
\hline Có & 266 & 67,3 & \\
\hline Không & 129 & 32,7 & Chưa từng đánh giá \\
\hline Tổng & 395 & 100,0 & \\
\hline
\end{tabular}

Nguồn: Tổng hợp từ kết quả khảo sát (2020)

Qua Bảng 1 cho thấy số lượng sinh viên trong mẫu khảo sát có tham gia đánh giá học phần trực tuyến ít nhất một môn trong thời gian theo học tại trường đến thời điểm hiện tại là 266 sinh viên, tương ứng $67,3 \%$. Số lượng sinh viên chưa từng tham gia đánh giá học phần trực tuyến chiếm $32,7 \%$. Qua số liệu này chứng tỏ sự tham gia rất hạn chế của sinh viên vào hoạt động đánh giá học phần.

\subsubsection{Các yếu tố nhân khẩu học của mẫu khảo sát}

\section{Bảng 2}

Các yếu tố nhân khẩu học của mẫu khảo sát

\begin{tabular}{|c|c|c|c|}
\hline \multicolumn{2}{|c|}{} & Số sinh viên & Tỷ lệ (\%) \\
\hline \multirow{3}{*}{ Giới tính } & Nam & 206 & 52,2 \\
\cline { 2 - 4 } & Nữ & 189 & 47,8 \\
\hline \multirow{3}{*}{ Dân tộc } & Kinh & 375 & 94,9 \\
\cline { 2 - 4 } & Hoa & 11 & 2,8 \\
\cline { 2 - 4 } & Khmer & 9 & 2,3 \\
\hline
\end{tabular}

Nguồn: Tổng hợp từ kết quả khảo sát (2020)

Qua Bảng 2 cho thấy, số lượng sinh viên nam tham gia vào mẫu khảo sát chiếm $52,2 \%$ và số lượng nữ tham gia vào mẫu khảo sát chiếm $47,8 \%$. Qua tỷ lệ này cho thấy không có sự chệnh lệch lớn về tỷ lệ sinh viên nam và nữ trong mẫu khảo sát. Ngoài ra, với phương pháp chọn mẫu ngẫu nhiên, đa số sinh viên được phỏng vấn là dân tộc Kinh chiếm 94,9\%, còn lại là dân tộc Hoa chiếm $2,8 \%$ và dân tộc Khmer với $2,3 \%$. Tuy có sự chênh lệch về số lượng sinh viên giữa các nhóm dân tộc nhưng số liệu này là hợp lý vì đa số sinh viên theo học tại trường là dân tộc Kinh, các nhóm dân tộc còn lại chỉ chiếm số lượng ít. 


\subsubsection{Kết quả học tập và kết quả rèn luyện của sinh viên trong mâu khảo sát}

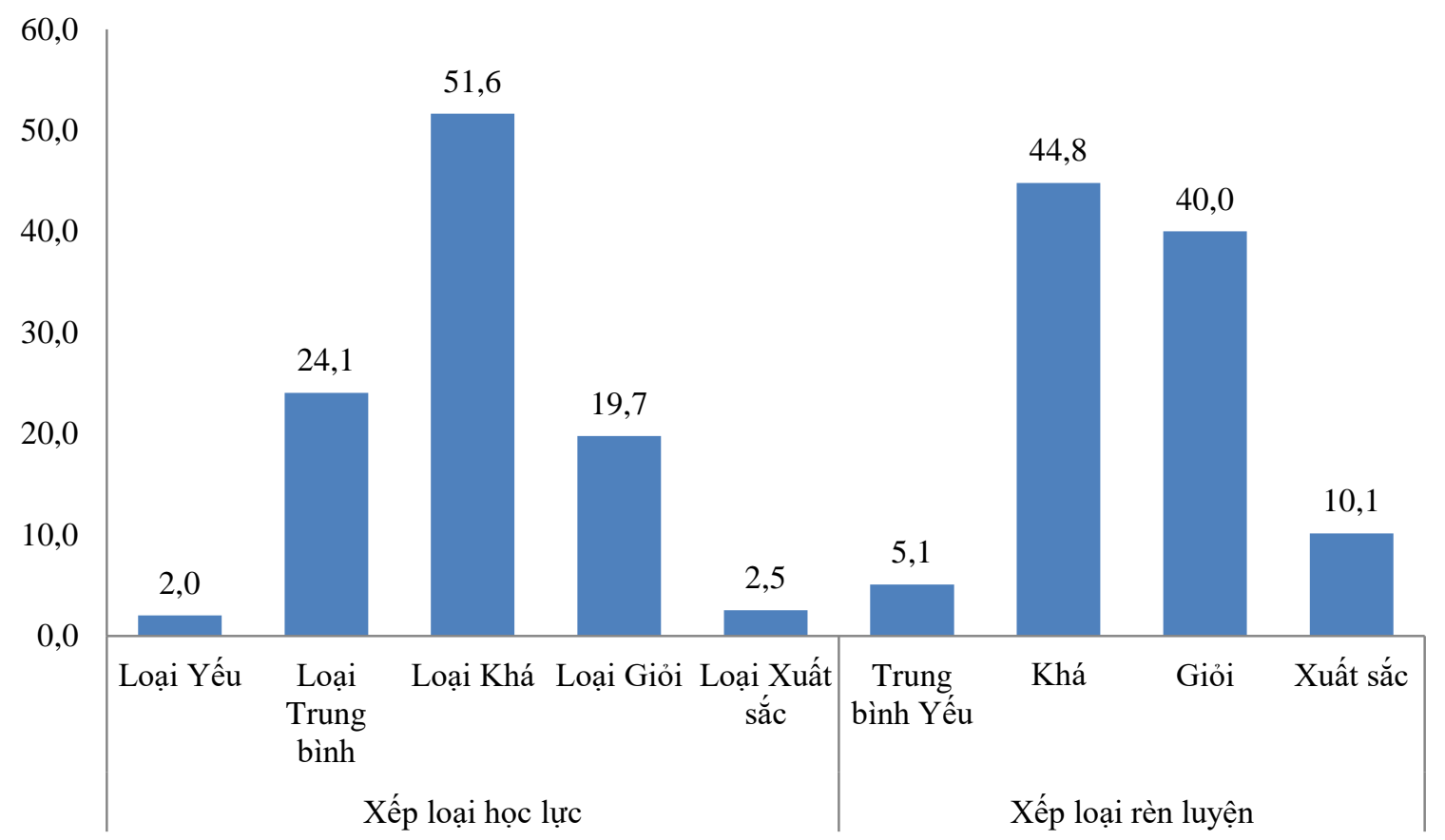

Hình 6. Kết quả học tập và kết quả rèn luyện của sinh viên

Nguồn: Tổng hợp từ kết quả khảo sát (2020)

Về mặt học lực, số lượng sinh viên trong mẫu khảo sát có điểm trung bình tích lũy đạt loại khá trở lên chiếm gần $74 \%$, còn lại là nhóm sinh viên có học lực trung bình - yếu chiếm gần $26 \%$. Qua đây cho thấy số lượng sinh viên yếu và trung bình chiếm hơn $1 / 4$ số lượng sinh viên trong mẫu khảo sát. Vì vậy, nhà trường cần có những biện pháp kịp thời hỗ trợ về phương pháp học tập giúp cải thiện học lực cho sinh viên các khóa.

Về mặt rèn luyện, đa số các sinh viên trong mẫu khảo sát có điểm rèn luyện đạt loại khá, giỏi với tỷ lệ gần $85 \%$, số lượng sinh viên đạt loại trung bình yếu chiếm tỷ lệ $5,1 \%$ và loại xuất sắc là $10,1 \%$. Đây cũng là một chỉ tiêu cần được xem xét khi đề xuất các giải pháp nhằm nâng cao tỷ lệ đánh giá học phần trong sinh viên hiện nay.

\subsubsection{Kiểm định mối liên hệ giũa quyết định đánh giá học phần và các biến nhân khẩu học}

Để kiểm định mối liên hệ giữa các biến định danh - định danh hoặc định danh - thứ bậc nghiên cứu sử dụng kiểm định Chi - bình phương để kiểm định mỗi liên hệ giữa quyết định tham gia đánh giá (gọi tắt là biến $\mathrm{Y}$ ) và các biến nhân khẩu học với giả thuyết:

$H_{O(i)}$ : Không có mối quan hệ giữa biến quyết định đánh giá và biến nhân khẩu học

$H_{1(i)}$ : Có mối quan hệ giữa biến quyết định đánh giá và các biến nhân khẩu học

Trong đó: i lần lượt là các biến nhân khẩu học được đưa vào mô hình kiểm định Chi bình phương, nghiên cứu lựa chọn mức ý nghĩa là $5 \%$.

Qua kiểm định Chi - bình phương nghiên cứu kết luận biến Khóa học, Kết quả rèn luyện, Trình độ tin học, Tình trạng sở hữu laptop có quan hệ với biến Y (độ tin cậy $95 \%$ ), các biến nhân khẩu học còn lại không có mối liên hệ với quyết định tham gia đánh giá. 


\subsubsection{Kiểm định Chi - bình phương giữa biến Y và biến khóa học}

\section{Bảng 3}

Kiểm định mối quan hệ giữa biến Y và biến khóa học

\begin{tabular}{|c|c|c|c|c|c|c|}
\hline & & & \multicolumn{3}{|c|}{ Khóa học } & \multirow{2}{*}{ Cộng } \\
\hline & & & K43 & K44 & K45 & \\
\hline \multirow{6}{*}{$\mathrm{Y}$} & \multirow{3}{*}{ Không } & Giá trị & 27,0 & 38,0 & 64,0 & 129,0 \\
\hline & & Giá trị kỳ vọng & 37,6 & 50,6 & 40,8 & 129,0 \\
\hline & & \% within Khóa học & $23,5 \%$ & $24,5 \%$ & $51,2 \%$ & $32,7 \%$ \\
\hline & \multirow{3}{*}{ Có } & Giá trị & 88,0 & 117,0 & 61,0 & 266,0 \\
\hline & & Giá trị kỳ vọng & 77,4 & 104,4 & 84,2 & 266,0 \\
\hline & & \% within Khóa học & $76,5 \%$ & $75,5 \%$ & $48,8 \%$ & $67,3 \%$ \\
\hline \multirow{3}{*}{\multicolumn{2}{|c|}{ Cộng }} & Giá trị & 115,0 & 155,0 & 125,0 & 395,0 \\
\hline & & Giá trị kỳ vọng & 115,0 & 155,0 & 125,0 & 395,0 \\
\hline & & \% within Khóa học & $100,0 \%$ & $100,0 \%$ & $100,0 \%$ & $100,0 \%$ \\
\hline
\end{tabular}

Nguồn: Kết quả được xử lý từ số liệu điều tra (2020)

Qua Bảng 3 cho thấy số lượng sinh viên không tham gia đánh giá học phần trực tuyến ở khóa 45 là khá cao so với các khóa còn lại. Điều này chứng tỏ các sinh viên khóa mới hơn chưa nắm thông tin về hoạt động đánh giá học phần trực tuyến. Vì vậy, nhà trường cần có những biện pháp kịp thời trong việc thông tin, hướng dẫn, triển khai nhằm giúp sinh viên khóa mới nắm được thông tin và tham gia đánh giá học phần kịp thời và đúng quy định.

\subsubsection{Kiểm định Chi - bình phuơng giũa biến Y và biến kết quả rèn luyện}

\section{Bảng 4}

Mối quan hệ giữa biến Y và biến kết quả rèn luyện

\begin{tabular}{|c|c|c|c|c|c|c|c|}
\hline & & & \multicolumn{4}{|c|}{ Xếp loại rèn luyện } & \multirow{2}{*}{ Cộng } \\
\hline & & & TB - Yếu & Khá & Giỏi & Xuất sắc & \\
\hline \multirow{6}{*}{$\mathrm{Y}$} & \multirow{3}{*}{ Không } & Giá trị & 11,0 & 65,0 & 42,0 & 11,0 & 129,0 \\
\hline & & Giá trị kỳ vọng & 6,5 & 57,8 & 51,6 & 13,1 & 129,0 \\
\hline & & \% within Xếp loại rèn luyện & $55,0 \%$ & $36,7 \%$ & $26,6 \%$ & $27,5 \%$ & $32,7 \%$ \\
\hline & \multirow{3}{*}{ Có } & Giá trị & 9,0 & 112,0 & 116,0 & 29,0 & 266,0 \\
\hline & & Giá trị kỳ vọng & 13,5 & 119,2 & 106,4 & 26,9 & 266,0 \\
\hline & & \% within Xếp loại rèn luyện & $45,0 \%$ & $63,3 \%$ & $73,4 \%$ & $72,5 \%$ & $67,3 \%$ \\
\hline \multirow{3}{*}{\multicolumn{2}{|c|}{ Cộng }} & Giá trị & 20,0 & 177,0 & 158,0 & 40,0 & 395,0 \\
\hline & & Giá trị kỳ vọng & 20,0 & 177,0 & 158,0 & 40,0 & 395,0 \\
\hline & & \% within Xếp loại rèn luyện & $100,0 \%$ & $100,0 \%$ & $100,0 \%$ & $100,0 \%$ & $100,0 \%$ \\
\hline
\end{tabular}

Nguồn: Kết quả được xử lý từ số liệu điều tra (2020) 
Qua Bảng 4 cho thấy số lượng sinh viên không tham gia đánh giá học phần trực tuyến có kết quả rèn luyện trung bình - yếu là khá cao chiếm $55 \%$, trong khi số lượng sinh viên có kết quả rèn luyện từ khá trở lên có tỷ lệ tham gia đanh giá học phần vượt trội, cụ thể là $63,3 \%$ đối với kết quả rèn luyện khá, $73,4 \%$ đối với kết quả rèn luyện giỏi và $72,5 \%$ đối với kết quả rèn luyện xuất sắc.

Từ số liệu ta dễ dàng kết luận, những sinh viên có điểm rèn luyện tốt thường tích cực tham gia vào các hoạt động. Ngược lại, các sinh viên có điểm rèn luyện yếu kém cần được nhà trường quan tâm nhiều hơn trong công tác tuyên truyền, khuyến khích tham gia lấy kiến đánh giá học phần nhằm cải thiện hoạt động đánh giá học phần trực tuyến tại trường.

\subsubsection{Kiểm định Chi - bình phương giữa biến Y và biến trình độ tin học}

Qua Bảng 5 cho thấy đa số các sinh viên trong mẫu khảo sát đều biết sử dụng và sử dụng thành thạo tin học. Đây là điều kiện thuận lợi để nhà trường áp dụng công nghệ thông tin vào tuyên truyền cũng như triển khai hoạt động đánh giá học phần trực tuyến trong thời gian tới.

\section{Bảng 5}

Mối quan hệ giữa biến Y và biến biến trình độ tin học

\begin{tabular}{|c|c|c|c|c|c|c|c|}
\hline & & & \multicolumn{4}{|c|}{ Trình độ tin học } & \multirow[b]{2}{*}{ Cộng } \\
\hline & & & $\begin{array}{l}\text { Chưa biết } \\
\text { sử dụng }\end{array}$ & $\begin{array}{c}\text { Biết sử dụng } \\
\text { nhưng không } \\
\text { thành thạo }\end{array}$ & $\begin{array}{c}\text { Biết sử } \\
\text { dụng }\end{array}$ & $\begin{array}{l}\text { Sử dụng } \\
\text { rất thành } \\
\text { thạo }\end{array}$ & \\
\hline \multirow{6}{*}{ Y } & \multirow{3}{*}{ Không } & Giá trị & 2,0 & 34,0 & 86,0 & 7,0 & 129,0 \\
\hline & & Giá trị kỳ vọng & 0,7 & 24,8 & 93,1 & 10,5 & 129,0 \\
\hline & & $\begin{array}{l}\text { \% within Trình } \\
\text { độ tin học }\end{array}$ & $100,0 \%$ & $44,7 \%$ & $30,2 \%$ & $21,9 \%$ & $32,7 \%$ \\
\hline & \multirow{3}{*}{ Có } & Giá trị & 0,0 & 42,0 & 199,0 & 25,0 & 266,0 \\
\hline & & Giá trị kỳ vọng & 1,3 & 51,2 & 191,9 & 21,5 & 266,0 \\
\hline & & $\begin{array}{l}\text { \% within Trình } \\
\text { độ tin học }\end{array}$ & $0,0 \%$ & $55,3 \%$ & $69,8 \%$ & $78,1 \%$ & $67,3 \%$ \\
\hline & \multirow{3}{*}{ Cộng } & Giá trị & 2,0 & 76,0 & 285,0 & 32,0 & 395,0 \\
\hline & & Giá trị kỳ vọng & 2,0 & 76,0 & 285,0 & 32,0 & 395,0 \\
\hline & & $\begin{array}{l}\text { \% within Trình } \\
\text { độ tin học }\end{array}$ & $100,0 \%$ & $100,0 \%$ & $100,0 \%$ & $100,0 \%$ & $100,0 \%$ \\
\hline
\end{tabular}

Nguồn: Kết quả được xử lý từ số liệu điều tra (2020) 


\subsubsection{Kiểm định Chi - bình phuoong giũa biến Y và biến có sở hũu laptop}

\section{Bảng 6}

Mối quan hệ giữa biến Y và biến biến có sở hữu laptop

\begin{tabular}{|c|c|c|c|c|c|}
\hline & & & \multicolumn{2}{|c|}{ Có sở hữu Laptop } & \multirow{2}{*}{ Cộng } \\
\hline & & & Không & Có & \\
\hline \multirow{6}{*}{$\mathrm{Y}$} & \multirow{3}{*}{ Không } & Giá trị & 39,0 & 90,0 & 129,0 \\
\hline & & Giá trị kỳ vọng & 26,1 & 102,9 & 129,0 \\
\hline & & \% within có sở hữu laptop & $48,8 \%$ & $28,6 \%$ & $32,7 \%$ \\
\hline & \multirow{3}{*}{ Có } & Giá trị & 41,0 & 225,0 & 266,0 \\
\hline & & Giá trị kỳ vọng & 53,9 & 212,1 & 266,0 \\
\hline & & \% within có sở hữu laptop & $51,3 \%$ & $71,4 \%$ & $67,3 \%$ \\
\hline \multirow{3}{*}{\multicolumn{2}{|c|}{ Cộng }} & Giá trị & 80,0 & 315,0 & 395,0 \\
\hline & & Giá trị kỳ vọng & 80,0 & 315,0 & 395,0 \\
\hline & & \% within có sở hữu laptop & $100,0 \%$ & $100,0 \%$ & $100,0 \%$ \\
\hline
\end{tabular}

Nguồn: Kết quả được xử lý từ số liệu điều tra (2020)

Qua Bảng 6 cho thấy tỷ lệ không tham gia đánh giá học phần trực tuyến ở nhóm không sở hữu laptop chiếm 48,8\% và tỷ lệ không tham gia đánh giá học phần trực tuyến ở nhóm có sở hữu laptop chiếm $28,6 \%$. Ngược lại, tỷ lệ có tham gia đánh giá học phần trực tuyến ở nhóm không sở hữu laptop chiếm $51,3 \%$ và tỷ lệ có tham gia đánh giá học phần trực tuyến ở nhóm có sở hữu laptop chiếm $71,4 \%$. Nghiên cứu kết luận những sinh viên có sở hữu laptop có xu hướng tham gia đánh giá học phần trực tuyến nhiều hơn. Thật vậy, sinh viên khi sở hữu laptop có nhiều thuận lợi trong việc kiểm tra thông tin, dễ dàng tiếp cận công nghệ và có công cụ thực hiện các yêu cầu từ phía nhà trường. Vì vậy, để cải thiện hoạt động đánh giá học phần trực tuyến nhà trường cần có những biện pháp như tăng cường phủ sóng mạng không dây để sinh viên có thể kết nối với máy tính cá nhân. Ngoài ra, đối với nhóm sinh viên không sở hữu laptop nhà trường cần hỗ trợ sinh viên tham gia đánh giá học phần bằng máy tính tại của nhà trường hay thông qua giao diện điện thoại thông minh có chức năng kết nối mạng không dây.

\subsubsection{Kiểm định Cronbach's Alpha}

Để đánh giá mức độ tương quan chặt chẽ giữa các biến quan sát trong cùng một nhân tố, nghiên cứu tiến hành kiểm định Cronbach's Alpha giữa các nhóm nhân tố. Kết quả xử lý số liệu điều tra cho thấy hệ số Cronbach' Alpha của các nhân tố đưa vào mô hình nghiên cứu đều lớn hơn 0,6 và dao động trong khoảng 0,740 đến 0,874 chứng tỏ các thang đo trong nghiên cứu này được đánh giá tốt và rất tốt. Xem xét hệ số tương quan biến tổng trong từng quan sát đều lớn hơn 0,3 và dao động trong khoảng 0,430 đến 0,786 ta kết luận các quan sát này đều đạt yêu cầu và được sử dụng để tiến hành phân tích nhân tố khám phá nhằm xem xét mối quan hệ giữa tất cả các nhân tố được đưa vào mô hình nghiên cứu.

\subsubsection{Phân tích nhân tố khám phá}

Qua Bảng 7, ta có hệ số KMO là $0,868>0,5$ chứng tỏ phân tích nhân tố khám phá là rất phù hợp đối với nghiên cứu. Ngoài ra, với giá trị Sig trong kiểm định Bartlett là 0,000 nhỏ hơn mức ý nghĩa $5 \%$, chứng tỏ các biến quan sát có tương quan với nhau trong tổng thể nghiên cứu. Căn cứ vào trị số Eigenvalue trong bảng tổng phương sai trích nghiên cứu xác định được năm 
nhóm nhân tố chính với giá trị tổng phương sai trích đạt $62,3 \%$, chứng tỏ năm nhân tốt được trích ra phản ánh được $62,3 \%$ sự biến thiên của dữ liệu của tất cả các biến quan sát được đưa vào mô hình.

\section{Bảng 7}

Kết quả ma trận nhân tố đã xoay

\begin{tabular}{|c|c|c|c|c|c|}
\hline & \multicolumn{5}{|c|}{ Nhóm nhân tố } \\
\hline & $\begin{array}{l}\text { Điều kiện } \\
\text { thuận lọi } \\
\text { được cung } \\
\text { cấp (F1) }\end{array}$ & $\begin{array}{c}\text { An toàn bảo } \\
\text { mật đối với } \\
\text { người dùng (F2) }\end{array}$ & $\begin{array}{c}\text { Môi } \\
\text { trường } \\
\text { xã hội } \\
\text { (F3) }\end{array}$ & $\begin{array}{c}\text { Cảm nhận về tính } \\
\text { dễ thực hiện } \\
\text { đánh giá (F4) }\end{array}$ & $\begin{array}{l}\text { Lọii ích có } \\
\text { được khi thực } \\
\text { hiện đánh giá } \\
\text { (F5) }\end{array}$ \\
\hline ĐKTL 2 & 0,744 & & & & \\
\hline ĐKTL 1 & 0,740 & & & & \\
\hline ĐKTL 4 & 0,687 & & & & \\
\hline ĐKTL 3 & 0,642 & & & & \\
\hline ĐKTL 5 & 0,585 & & & & \\
\hline ĐKTL 6 & 0,524 & & & & \\
\hline ATBM 2 & & 0,858 & & & \\
\hline ATBM 3 & & 0,819 & & & \\
\hline ATBM 1 & & 0,816 & & & \\
\hline ATBM 4 & & 0,657 & & & \\
\hline AHXH 3 & & & 0,883 & & \\
\hline AHXH 4 & & & 0,841 & & \\
\hline AHXH 2 & & & 0,833 & & \\
\hline AHXH 1 & & & 0,647 & & \\
\hline DTH 1 & & & & 0,818 & \\
\hline DTH 2 & & & & 0,791 & \\
\hline DTH 3 & & & & 0,647 & \\
\hline DTH 4 & & & & 0,481 & \\
\hline LI 2 & & & & & 0,775 \\
\hline LI 4 & & & & & 0,759 \\
\hline LI 1 & & & & & 0,719 \\
\hline LI 3 & & & & & 0,495 \\
\hline \multicolumn{5}{|c|}{ Hệ số KMO } & 0,868 \\
\hline \multicolumn{5}{|c|}{ Sig. Kiểm định } & 0,000 \\
\hline \multicolumn{5}{|c|}{ Barlette's } & $3.784,785$ \\
\hline \multicolumn{5}{|c|}{ Eigenvalue } & 1,192 \\
\hline \multicolumn{5}{|c|}{ Phương sai trích } & $62,272 \%$ \\
\hline
\end{tabular}


Nguồn: Kết quả được xử lý từ số liệu điều tra (2020)

\subsubsection{Phân tích mô hình hồi quy logistic}

Nhằm củng cố và đề xuất các giải pháp tăng cường quyết định tham gia lấy ý kiến đánh giá học phần của sinh viên tại trường Đại học Cần Thơ, nghiên cứu tiến hành phân tích hồi quy logistic với kết quả được xử lý từ số liệu điều tra như sau:

\section{Bảng 8}

Kết quả mô hình hồi quy logistic

\begin{tabular}{|c|c|c|c|c|c|c|c|}
\hline Biến độc lập & Mã hóa lại biến² & B & S.E. & Wald & df & Sig. & $\operatorname{Exp}(B)$ \\
\hline Giới tính & DGioitinh &, 273 & ,289 & 894 & 1 &, 344 & 1,314 \\
\hline \multirow{2}{*}{ Dân tộc } & DKinh &,- 596 &, 852 & ,490 & 1 & ,484 &, 551 \\
\hline & DHoa &,- 770 & 1,062 &, 526 & 1 & ,468 & ,463 \\
\hline \multirow{2}{*}{ Nơi ở } & DKTX &, 250 & ,392 & 407 & 1 & ,524 & 1,284 \\
\hline & DNhatro & ,063 & 312 & 040 & 1 &, 841 & 1,065 \\
\hline \multirow{2}{*}{$\underline{\text { Khóa hoc }}$} & $\underline{D K 43^{* * *}}$ & $\underline{1,006}$ & .332 & $\underline{9,200}$ & $\underline{1}$ & .002 & $\underline{2,735}$ \\
\hline & $\underline{D K 44^{* * *}}$ & $\underline{1,057}$ &, 311 & $\underline{11,546}$ & $\underline{1}$ & .001 & $\underline{2,877}$ \\
\hline \multirow{2}{*}{ Khoa đào tạo } & DKinhte &, 051 & ,367 & ,019 & 1 & ,889 & 1,052 \\
\hline & DCongnghe &,- 253 &, 351 & ,519 & 1 & 471 & ,776 \\
\hline \multirow{4}{*}{$\begin{array}{c}\text { Kết quả học } \\
\text { tập }\end{array}$} & DYeu & 1,700 & 1,574 & 1,167 & 1 & ,280 & 5,472 \\
\hline & \begin{tabular}{|l} 
DTrungbinh \\
\end{tabular} &,- 783 & ,952 &, 677 & 1 & ,411 &, 457 \\
\hline & DKha &,- 866 & ,907 & ,911 & 1 & 340 & ,421 \\
\hline & DGioi &,- 843 & 917 & ,846 & 1 & ,358 & 430 \\
\hline \multirow{3}{*}{$\begin{array}{c}\text { Kết quả rèn } \\
\text { luyện }\end{array}$} & DRTrungbinhYeu &,- 443 &, 728 & ,371 & 1 & ,543 & ,642 \\
\hline & DRLKha &, 038 & ,505 & ,006 & 1 & ,940 & 1,039 \\
\hline & DRLGioi & ,395 & ,491 & ,649 & 1 & 421 & 1,485 \\
\hline \multirow{3}{*}{$\begin{array}{c}\text { Trình độ tin } \\
\text { học }\end{array}$} & Dchuabiet & $-22,333$ & 27314,777 &, 000 & 1 & ,999 &, 000 \\
\hline & Dbietkothao &,- 594 &, 583 & 1,038 & 1 & ,308 &, 552 \\
\hline & Dbietsudung &,- 274 &, 526 & 271 & 1 & 603 & ,761 \\
\hline \multirow{3}{*}{$\begin{array}{l}\text { Loại điện } \\
\text { thoại đang } \\
\text { dùng }\end{array}$} & Dnghegoi &,- 282 & 1,392 &, 041 & 1 &, 840 &, 755 \\
\hline & DSmartphoneko3G &, 430 &, 685 & ,394 & 1 &, 530 & 1,537 \\
\hline & DSmartphone $3 \mathrm{G}$ &,- 180 & 652 & ,076 & 1 &, 783 &, 835 \\
\hline \multirow{3}{*}{$\begin{array}{l}\text { Công cụ truy } \\
\text { cập internet }\end{array}$} & DDienthoai &,- 510 & 1,079 & ,223 & 1 & ,637 & ,601 \\
\hline & DLaptop &,- 435 & 1,149 & , 143 & 1 & ,705 &, 647 \\
\hline & DMaytinhtruong & 19,423 & 16989,808 &, 000 & 1 & ,999 & 272507623, \\
\hline
\end{tabular}

${ }^{2}$ Các biến với chữ cái đầu tiên là "D" là biến Dummy, ví dụ như DKinh, DHoa là biến giả được mã hóa lại nhằm phục vụ cho phân tích mô hình hồi quy logistic. 


\begin{tabular}{|c|c|c|c|c|c|c|c|}
\hline Biến độc lập & Mã hóa lại biến² & B & S.E. & Wald & df & Sig. & $\operatorname{Exp}(B)$ \\
\hline & & & & & & & 847 \\
\hline $\begin{array}{l}\text { Tình trạng } \\
\text { làm thêm }\end{array}$ & DLamthem &,- 237 & ,268 &, 782 & 1 & ,376 & ,789 \\
\hline $\begin{array}{c}\text { Tình trạng sở } \\
\text { hữu laptop }\end{array}$ & DLaptop & ,435 &, 327 & 1,772 & 1 &, 183 & 1,545 \\
\hline $\mathrm{F} 1$ & ĐKTL &, 131 &, 129 & 1,043 & 1 & ,307 & 1,140 \\
\hline$\underline{F 2}$ & $\underline{A T B M^{* * *}}$ &, 344 &, 125 & $\underline{7,514}$ & $\underline{1}$ &, 006 & $\underline{1,410}$ \\
\hline $\mathrm{F} 3$ & MTXH & ,200 &, 136 & 2,177 & 1 &, 140 & 1,222 \\
\hline$\underline{F 4}$ & $\underline{D T H^{* * *}}$ &, 564 &, 135 & $\underline{17,463}$ & $\underline{1}$ &, 000 & $\underline{1,758}$ \\
\hline \multirow[t]{2}{*}{$\underline{F 5}$} & $\underline{L I * *}$ &, 286 &, 129 & $\underline{4,905}$ & $\underline{1}$ & .027 & $\underline{1,331}$ \\
\hline & Constant & 1,798 & 1,731 & 1,079 & 1 & ,299 & 6,039 \\
\hline \multicolumn{7}{|c|}{ Sig. (Omnibus Tests of Model Coefficients) } & 0,000 \\
\hline \multicolumn{7}{|c|}{-2 Log likelihood } & 410,061 \\
\hline \multicolumn{7}{|c|}{ Pseudo $\mathrm{R}^{2}$} & 0,281 \\
\hline \multicolumn{7}{|c|}{ Hosmer and Lemeshow Test } & 0,948 \\
\hline \multicolumn{7}{|c|}{ PAC (Percentage Accuracy in Classification) } & $74,2 \%$ \\
\hline
\end{tabular}

Nguồn: Kết quả được xử lý từ số liệu điều tra (2020)

Từ kết quả mô hình hồi quy logistic, ta có giá trị Sig trong kiểm định Omnibus Tests of Model Coefficients đều bằng $0,000<0,05$ chứng tỏ mô hình có ý nghĩa thống kê với độ tin cậy $95 \%$. Các chỉ tiêu như -2 Log likelihood, $\mathrm{R}^{2}$, Hosmer and Lemeshow Test trong kết quả mô hình hồi quy logistic chỉ mang ý nghĩa kiểm tra sự phù hợp giữa các mô hình khác nhau khi tiến hành loại các biến có giá trị Sig lớn trong kiểm định Wald chứ không mang ý nghĩa đánh giá độ tin cậy của mô hình như các hàm hồi quy khác. Giá trị $\mathrm{PAC}$ của mô hình bằng $74,2 \%$ chứng tỏ tỷ lệ mô hình ước lượng chính xác lên đến $74,2 \%$. Từ kết quả ở Bảng 8 , ta có phương trình hồi quy Logistic được viết như sau:

$$
\log \left(\frac{p}{1-p}\right)=1,798+0,273 \text { DGioitinh }-0,596 \text { DKinh }-0,770 \text { DHoa }+0,250 \text { DKTX }+
$$
0,063 DNhatro $+1,006 D K 43^{* * *}+1,057 D K 43^{* * *}+0,051$ DKinhte $-0,253$ DCongnghe + 1,700DYeu - 0,783DTrungbinh -0,866DKha-0,843DGioi - 0,443DRLTrungbinhYeu + 0,038DRLKha +0,395DRLGioi - 22,333Dchuabiet - 0,594Dbietkothao -

0,274Dbietsudung - 0,282Dnghegoi $+0,430 D$ Smartphoneko3G-0,180DSmartphone $3 G-$ 0,510Dienthoai - 0,435DLaptop + 19,423DMaytinhtruong - 0,237DLamthem + 0,435 L Laptop $+0,131 Đ K T L+0,344 A T B M^{* * *}+0,200 M T X H+0,564 D T H^{* * *}+0,286 L I^{* *}$

Từ phương trình hồi quy ta kết luận có ba nhân tố tác động đến quyết định tham gia lấy ý kiến đánh giá học phần của sinh viên Đại học Cần Thơ, cụ thể:

Với giá trị $\mathrm{P}_{\text {value }}$ của nhân tố “An toàn bảo mật đối với người dùng” là 0,006 nhỏ hơn mức ý nghĩa $1 \%$, ta bác bỏ giả thuyết $\mathrm{H}_{0(5)}$ và chấp nhận giả thuyết $\mathrm{H}_{1(5)}$ : An toàn bảo mật đối với người dùng có ảnh hưởng tích cực làm tăng xác suất quyết định thực hiện đánh giá học phần của sinh viên. 
Với giá trị $\mathrm{P}_{\text {value }}$ của nhân tố "Cảm nhận về tính dễ thực hiện đánh giá” là 0,000 nhỏ hơn mức ý nghĩa $1 \%$, ta bác bỏ giả thuyết $\mathrm{H}_{0(2)}$ và chấp nhận giả thuyết $\mathrm{H}_{1(2)}$ : Cảm nhận về tính dễ thực hiện đánh giá có ảnh hưởng tích cực làm tăng xác suất đến quyết định thực hiện đánh giá học phần của sinh viên.

Với giá trị $\mathrm{P}_{\text {value }}$ của nhân tố "Lợi ích có được khi thực hiện đánh giá” 0,027 nhỏ hơn mức ý nghĩa $5 \%$, ta bác bỏ giả thuyết $\mathrm{H}_{0(1)}$ và chấp nhận giả thuyết $\mathrm{H}_{1(1)}$ : Lợi ích có được khi thực hiện đánh giá có ảnh hưởng tích cực làm tăng xác suất quyết định thực hiện đánh giá học phần của sinh viên.

Trong các biến kiểm soát về nhân khẩu học được nghiên cứu đề xuất, biến khóa học có ý nghĩa thống kê ở mức ý nghĩa $1 \%$, chứng tỏ những sinh viên khóa cũ có xác suất thực hiện đánh giá học phần cao hơn sinh viên khóa mới.

Các nhân tố còn lại tuy có ý nghĩa với các nghiên cứu đi trước, nhưng xem xét tại trường Đại học Cần Thơ thì giá trị Sig trong kiểm định Wald đều lớn hơn 0,1 , ta kết luận các nhân tố như ĐKTL và MTXH không ảnh hưởng đến quyết định tham gia đánh giá học phần của sinh viên.

\subsection{Thảo luận chung}

Với mục tiêu tìm ra các nhân tố ảnh hưởng đến quyết định tham gia lấy ý kiến đánh giá học phần của sinh viên và đề xuất các giải pháp nhằm cải thiện hoạt động này. Nghiên cứu kết luận Khóa học có ảnh hưởng đến quyết định đánh giá học phần trực tuyến của sinh viên. Cụ thể, nhóm sinh viên năm thứ ba và năm thứ tư (lần lượt là sinh viên Khóa 44, sinh viên Khóa 43) sẽ có đủ thông tin và kỹ năng để tham gia đánh giá học phần trực tuyến. Ngược lại, nhóm sinh viên năm một (khóa 46) và năm hai (khóa 45) là những tân sinh viên với thời gian theo học tại trường ngắn, được nhà trường ấn định thời khóa biểu, phân nhóm học phần cố định, đồng thời chưa có nhiều thông tin về hoạt động đánh giá học phần, dẫn đến quá hạn thực hiện đánh giá hoặc không có thông tin để tham gia đánh giá. Vì vậy, nhà trường cần dành thời gian và nguồn lực để cải thiện tình trạng tham gia đánh giá học phần của sinh viên khóa mới.

Đối với nhân tố an toàn bảo mật cho người dùng, các nghiên cứu đi trước đã khẳng định đây là nhóm nhân tố có vai trò rất quan trọng ảnh hưởng đến hành vi tham gia đánh giá học phần của sinh viên. Nhưng xem xét tại trường Đại học Cần Thơ, sinh viên phải dùng mã số sinh viên đăng nhập vào tài khoản cá nhân mới có thể thực hiện đánh giá học phần. Chính điều này một phần nào cản trở sinh viên tham gia đánh giá học phần. Vì vậy, việc làm rõ cho sinh viên nắm được tính ẩn danh, bảo mật của hệ thống đánh giá là rất quan trọng, từ đó làm tăng xác suất tham gia đánh giá học phần của sinh viên.

Ngoài ra, "nhân tố cảm nhận về tính dễ thực hiện đánh giá" (DTH) có tác động tích cực làm tăng xác suất quyết định tham gia tham gia đánh giá học phần với mức ý nghĩa $1 \%$. Tuy nhiên, đối với trường Đại học Cần Thơ, việc xây dựng hệ thống đánh giá trực tuyến còn ghi nhận điểm bất cập và gây khó khăn cho sinh viên. Cụ thể, để vào được hệ thống đánh giá sinh viên phải trải qua 2 bước: bước 01 truy cập vào giao diện tài khoản cá nhân, từ đây sinh viên phải đăng nhập vào một website khác để có thể thực hiện đánh giá. Cách bố trí này gây khó khăn cho sinh viên trong quá trình tìm hiểu và thực hiện đánh giá học phần. Vì vậy, việc xem xét thiết lập lại giao diện hệ thống đánh giá học phần là cần thiết.

Cuối cùng, quyết định đánh giá học phần chịu sự tác động của nhân tố "lợi ích có được khi thực hiện đánh giá”, với mức ý nghĩa $5 \%$. Rất nhiều sinh viên trong quá trình trả lời khảo sát chưa nắm được ý nghĩa của hoạt động này, một phần xuất phát từ cá nhân sinh viên, một phần bị chi phối bởi tư tưởng của giảng viên cho rằng sinh viên tham gia đánh giá không giúp ít gì cho 
họ trong quá trình giảng dạy. Chính điều này đã gây ra các suy nghĩ tiêu cực trong sinh viên về các lợi ích của hoạt động đánh giá học học phần. Ngoài ra, kết quả đánh giá chỉ dùng cho công tác kiểm định chất lượng và sinh viên không được cung cấp kết quả chung cho mỗi đợt lấy ý kiến. Vì vậy, việc tuyên tuyền làm rõ cũng như cung cấp kết quả đánh giá cho sinh viên có ý nghĩa quan trọng nhằm cải thiện hoạt động này.

\subsection{Một số giải pháp nhằm cải thiện công tác lấy ý kiến của sinh viên}

Đối với sinh viên khóa mới nhà trường nên tăng cường công tác hướng dẫn sinh viên về hoạt động đánh giá học phần trực tuyến. Xây dựng các video hướng dẫn giúp sinh viên có cái nhìn trực quan hơn. Quy định rõ trách nhiệm của giảng viên về việc nhắc nhở và hỗ trợ sinh viên thực hiện đánh giá. Sinh viên năm 1 có lịch học cố định và tập trung nên giảng viên có thể dành thời gian tại lớp để sinh viên tham gia đánh giá kịp thời.

Tăng cường bảo mật thông tin nhằm giúp sinh viên tự tin tham gia và thể hiện quan điểm. Nhà trường có thể tổ chức lấy ý kiến theo nhóm học phần thông qua hệ thống E-learning của từng học phần mà không cần đăng nhập vào hệ thống quản lý đào tạo. Làm rõ cho sinh viên về tính bảo mật của hệ thống đánh giá học phần trực tuyến của trường.

Cần giúp sinh viên cảm nhận về tính dễ thực hiện của hệ thống lấy ý kiến trực tuyến qua việc xây dựng lại giao diện truy cập vào hệ thống đánh giá học phần, thay vì sinh viên phải thực hiện nhiều bước mới có thể tham gia đánh giá. Trong dài hạn, nhà trường cần xem xét xây dựng một ứng dụng trên điện thoại nhằm tích hợp tất cả các hoạt động và có chức năng tự động nhắc nhở sinh viên chưa hoàn thành đánh giá học phần. Đa dạng về thời gian thực hiện đánh giá và tăng cường phủ sóng mạng không dây tại các nhà học để sinh viên có thực hiện đánh giá học phần tại lớp.

Tăng cường truyền thông về lợi ích của hoạt động đánh giá học phần, nhà trường và giảng viên cần làm rõ cho sinh viên hiểu được lợi ích của hoạt động đánh giá học phần trực tuyến qua việc cung cấp kết quả đánh giá học phần cho sinh viên, hoặc cung cấp thông tin về các cải tiến, các sáng kiến mới được áp dụng dựa trên kết quả đánh giá học phần trực tuyến.

\section{Kết luận}

Công tác lấy ý kiến sinh viên về hoạt động giảng dạy của giảng viên là một công tác quan trọng nhằm nâng cao chất lượng GDĐH. Hoạt động này đã được Đại học Cần Thơ triển khai nhiều năm nhưng chưa đạt được hiệu quả. Qua số liệu và phân tích, nghiên cứu kết luận để tăng xác suất tham gia đánh giá học phần trong sinh viên thì nhà trường cần đẩy mạnh công tác truyền thông nhằm nâng cao nhận thức trong sinh viên về hoạt động đánh giá học phần trực tuyến. Cần làm rõ cho sinh viên biết về các giá trị mà đánh giá học phần trực tuyến mang lại. Tuyên truyền để sinh viên biết về tính bảo mật của hệ thống đánh giá học phần trực tuyến và xây dựng lại giao diện đánh giá học phần dễ sử dụng hơn. Đa dạng khung thời gian để các sinh viên chưa tham gia đánh giá có thể thực hiện bổ sung nhằm cung cấp số liệu đầy đủ và chính xác giúp nhà trường nâng cao chất lượng đào tạo.

\section{Tài liệu tham khảo}

Adams, M. J. D. (2010). No evaluation left behind: Nonresponse in online course evaluations (Doctoral dissertation). North Carolina State University, Raleigh.

Ajzen, I. (1991). The theory of planned behavior. Organizational Behavior and Human Decision Processes, 50(2), 179-221. doi:10.1016/0749-5978(91)90020-T 
Avery, R. J., Bryant, W. K., Mathios, A., Kang, H., \& Bell, D. (2006). Electronic course evaluations: Does an online delivery system influence student evaluations? Journal of Economic Education, 37(1), 21-37. doi:10.3200/JECE.37.1.21-37

Ballantyne, C. (2003). Online evaluations of teaching: An examination of current practice and considerations for the future. New Directions for Teaching and Learning, 96, 103-112. doi:10.1002/tl.127

Bộ Giáo dục và Đào tạo. (2008). Hướng dẫn tổ chức lấy ý kiến phản hồi tù nguời học về hoạt động giảng dạy của giảng viên [Guide the organization to get feedback from learners about teaching activities of lecturers]. Retrieved March 20, 2020, from https://hethongphapluat.com/cong-van-1276-bgddt-ng-huong-dan-to-chuc-lay-y-kienphan-hoi-tu-nguoi-hoc-ve-hoat-dong-giang-day-cua-giang-vien-do-bo-giao-duc-va-daotao-ban-hanh.html

Bộ Giáo dục và Đào tạo. (2012). Quy định điều kiện thành lập và giải thể, nhiệm vu, quyền hạn của tổ chức kiểm định chất lương giáo dục [To provide for the establishment and dissolution conditions, the tasks and powers of the education quality accreditation organization]. Retrieved March 20, 2020, from https://hethongphapluat.com/thong-tu-612012-tt-bgddt-ve-quy-dinh-dieu-kien-thanh-lap-va-giai-the-nhiem-vu-quyen-han-cua-tochuc-kiem-dinh-chat-luong-giao-duc-do-bo-truong-bo-giao-duc-va-dao-tao-ban-hanh.html

Bộ Giáo dục và Đào tạo. (2012). Thông tu 61/TT-BGDĐT ngày 28 tháng 12 năm 2012 ban hành quy định điều kiện thành lập và giải thể, nhiệm vụ, quyền hạn của tổ chúc kiểm định chất luợng giáo duc [Circular 61/TT-BGDĐT dated 28 December 2012 promulgate the regulations on the establishment and dissolution conditions, the tasks and powers of education quality accreditation organizations]. Retrieved March 20, 2020, from https://thuvienphapluat.vn/van-ban/giao-duc/Thong-tu-61-2012-TT-BGDDT-dieu-kienthanh-lap-giai-the-nhiem-vu-quyen-han-163086.aspx

Campbell, C., \& Wende, M. V. (2000). International initiatives and trends in quality assurance for european higher education: European network of quality assurance agencies. Retrieved March 16, 2020, from https://www.enqa.eu/wp-content/uploads/initiatives.pdf

Cheng, Y. T., \& Tam, W. M. (1997). Multi-models of quality in education. Quality Assurance in Education, 5(1), 22-31.

Cummings, R., \& Ballatyne, C. (1999). Student feedback on teaching: Online! On target? Retrieved March 15, 2020, from https://www.researchgate.net/publication/242065066_ Student_Feedback_on_Teaching_Online_On_target

Dommeyer, C. J., Baum, P., Hanna, R. W., \& Chapman, K. S. (2004). Gathering faculty teaching evaluations by in-class and online surveys : Their effects on response rates and evaluations. Assessment \& Evaluation in Higher Education, 29(5), 611-623. doi:10.1080/02602930410001689171

Eng, T. H., Ibrahim, A. F., \& Shamsuddin, N. E. (2015). Students' perception: Student Feedback Online (SuFO) in higher education. Procedia - Social and Behavioral Sciences, 167, 109116. doi:10.1016/j.sbspro.2014.12.651

Fan, J., \& Yan, Z. (2010). Factors affecting response rates of the web survey: A systematic review. Computers in Human Behavior journal, 26(2), 132-139. doi:10.1016/j.chb.2009.10.015 
Fike, D. S., Doyle, D. J., \& Connelly, R. J. (2010). Online vs. paper evaluations of faculty: When less is good. The Journal of Effective Teaching, 10(2), 42-54.

Fishbein, M., \& Ajzen, I. (1981). Attitudes and voting behavior: An application of the theory of reasoned action. In G. M. Stephenson \& J. M. Davis (Eds.), Progress in applied social psychology (pp. 253-313). London, UK: Wiley.

Franklin, J., \& Theall, M. (1992). Disciplinary differences: Instructional goals and activities, measures of student performance, and student ratings of instruction. Retrieved March 10, 2020, from https://eric.ed.gov/?id=ED346786.

Goodman, J., Anson, R., \& Belcheirc, M. (2015). The effect of incentives and other instructordriven strategies to increase online student evaluation response rates. Assessment \& Evaluation in Higher Education, 40(7), 958-970. doi:10.1080/02602938.2014.960364

Harvey, L., \& Knight, P. T. (1996). Transforming higher education. In Digital agency in higher education (pp. 3-17). doi:10.4324/9780429020629-2

Hoang, T., \& Chu, N. N. M. (2008). Phân tích dĩ liệu nghiên cứu với SPSS [Analyze research data with SPSS]. Hanoi, Vietnam: Nhà xuất bản Hồng Đức.

Nair, S., Commission, T. E., \& Mertova, P. (2015). Student engagement: The key to improving survey response rates. Quality in Higher Education, 24(3), 225-232. doi:10.1080/13538320802507505

Nguyen, D. T. (2013). Phương pháp nghiên cúu khoa học trong kinh doanh [Scientific research method in business]. Hanoi, Vietnam: Nhà xuất bản Tài chính.

Nguyen, K. D., \& Pham, X. T. (2003). Về một số khái niệm thường dùng trong đảm bảo chất luọng giáo duc đại hoc [About some commonly used concepts in quality assurance of higher education]. Retrieved March 20, 2020, from https://tailieu.vn/doc/ve-mot-so-khainiem-thuong-dung-trong-dam-bao-chat-luong-giao-duc-dai-hoc-154147.html

Norris, D. F., \& Moon, M. J. (2005). Advancing e-government at the grassroots: Tortoise or hare? Public Administration Review, 65(1), 64-75. doi:10.1111/j.1540-6210.2005.00431

Nowell, C., Gale, L. R., \& Handley, B. (2010). assessing faculty performance using student evaluations of teaching in an uncontrolled setting. Assessment and Evaluation in Higher Education, 35(4), 463-475. doi:10.1080/02602930902862875

Porter, S. R., \& Umbach, P. D. (2006). Student survey response rates across institutions: Why Do they vary? Research in Higher Education, 47(2), 229-247. doi:10.1007/s11162-0058887-1

Ravelli, B. (2000). Anonymous online teaching assessments: Preliminary findings. Paper presented at Annual National Conference of the American Association for Higher Education, Charlotte, North Carolina.

Rogers, E. M. (1995), Diffusion of innovations (4th ed.). New York, NY: Free Press.

Ryan, P. (2015). Quality assurance in higher education: A review of literature. Higher Learning Research Communications, 5(4), 1-12. doi:10.18870/hlrc.v5i4.257

Sax, L. J., Gilmartin, S. K., \& Bryant, A. N. (2011). Assessing response rates and nonresponse bias in web. Higher Education, 44(4), 409-432. 
Schindler, L., Puls-Elvidge, S., Welzant, H., \& Crawford, L. (2015). definitions of quality in higher education: A synthesis of the literature. Higher Learning Research Communications, 5(3), 3-13. doi:10.18870/hlrc.v5i3.244

Spencer, K. J., \& Schmelkin, L. P. (2010). Student perspectives on teaching and its evaluation. Assessment \& Evaluation in Higher Education, 27(5), 397-409. doi:10.1080/0260293022000009285

Standish, T., Joines, J. A., Young, K. R., \& Gallagher, V. J. (2018). Improving SET response rates: Synchronous online administration as a tool to improve evaluation quality. Research in Higher Education, 59, 812-823. doi:10.1007/s11162-017-9488-5

Trung tâm quản lý chất lượng. (2020). Báo cáo thống kê công tác lấy ý kiến của các bên liên quan năm 2020 [Statistical report on stakeholder consultation in 2020]. Can Tho, Vietnam: Trường Đại học Cần Thơ.

Ubong, B., \& Okpor, M. O. (2019). Student Assessment of Teachers (SAT): Towards a basket of approaches. Asian Journal of University Education, 15(2), 79-94. doi:10.24191/ajue.v15i2.7558

Venkatesh, V., Morris, M. G., Davis, G. B., \& Davis, F. D. (2003). User acceptance of information technology: Toward a unified view. MIS Quarterly, 27(3), 425-478. doi: $10.2307 / 30036540$

Young, K., Joines, J., Standish, T., \& Gallagher, V. (2017). Student evaluations of teaching: The impact of faculty procedures on response rates. Assessment \& Evaluation in Higher Education, 44(1), 37-49. doi:10.1080/02602938.2018.1467878 